\title{
Identification, Isolation and Detection of Metallo Beta Lactamase Resistance in Acinetobacter Species from Various Clinical Samples in a Tertiary Care Hospital
}

\author{
Sundararajan Thangavel, Gomathi Manian* and Neelaveni Ramasamy \\ Department of Microbiology, Govt. Mohan Kumaramangalam Medical College, \\ Salem, Tamil Nadu, India \\ *Corresponding author
}

\begin{tabular}{|l|}
\hline Ke y w o r d s \\
Acinetobacter, Metallo \\
Beta Lactamase \\
(MBL), Combined \\
Disc Diffusion Test \\
(CDDT),
\end{tabular}

\section{Keywords}

BL), Combined

\section{A B S T R A C T}

Non fermenting Gram Negative Bacilli (NFGNB) once considered as contaminants, now emerged as a major cause of life threatening nosocomial infections and as multidrug resistant pathogens. Acinetobacter species are the opportunistic pathogens with increasing prevalance in the nosocomial infections. Community acquired infections are also common in Acinetobacter. It accounts for $10 \%$ of all community-acquired bacteremic pneumonias.

To isolate, identify and detect Carbapenem resistance producing Acinetobacter spp., and confirm Metallo Beta Lactamase ( MBL) production by phenotypic methods. This cross sectional study conducted in a tertiary care hospital for a period of 6 months from various clinical samples were identified using standard protocol. The MBL resistant strains of Acinetobacter species were identified by Kirby-Bauer disc diffusion methods and confirmed by phenotypic methods. Out of clinically significant isolates of Acinetobacter spp., 50 (67\%) were Acinetobacter baumannii and 25 (33\%) were Acinetobacter lwoffi. The antimicrobial susceptibility pattern revealed maximum resistance to Gentamycin (64\%), Cotrimoxazole, Amikacin \& Ciprofloxacin (40\%) and Cefotaxime and Ceftazidime (36\%). Sensitivity to Polymyxin B (100\%) followed by Imipenem and Meropenem (90\%). Among them 7 (9.3\%.) isolates were MBL producers. Among the 7 isolates, CDDT was positive in $5(71 \%)$ isolates, DDST was positive in 3(43\%) isolates. Acinetobacter baumannii were the most common isolate in this study. Difference in antimicrobial susceptibility poses a great problem in treating these infections. MBL production by these organisms leads to high morbidity and mortality and left with the only option of treating them by potentially toxic drugs like Colistin and Polymyxin B.

\section{Introduction}

Non Fermenting Gram Negative Bacilli (NFGNB) are aerobic, non-spore forming organisms that do not utilize carbohydrates as a source of energy (or) degrade them through metabolic pathways other than fermentation $(1,2,3)$. These are ubiquitous in nature and frequently considered as contaminants, most of them have emerged as important nosocomial pathogens causing opportunistic infections which account for about $15 \%$ of all 
bacterial isolates from a clinical microbiology laboratory ${ }^{(3)}$ This group includes organisms from genera like Pseudomonas, Acinetobacter, Stenotrophomonas, Burkholderia, Alcaligenes, Weeksella and many more. Currently, Pseudomonas aeruginosa and Acinetobacter baumanii are the most commonly isolated nonfermenters pathogenic for humans whereas infections caused by other species are relatively infrequent ${ }^{(4)}$.

Acinetobacter species are the opportunistic pathogens with increasing prevalence in the nosocomial infections (5). It accounts for $10 \%$ of all community-acquired bacteremic pneumonias ${ }^{(6)}$. Acinetobacter spp., have been reported to cause high mortality rate of $32 \%$ to $52 \%$ in blood stream infections. Similarly mortality rate up to $70 \%$ have been reported in ICU acquired pneumonia ${ }^{(7)}$. Different Acinetobacter species have differences in their antimicrobial susceptibility pattern, hence it is important to identify Acinetobacter isolates at species level ${ }^{(8)}$. A. baumannii is the most common species isolated from clinical specimens and they developed $70 \%$ of resistance to third generation cephalosporins, aminoglycosides and quinolones. $87 \%$ of Acinetobacter isolates were Multidrug resistant ${ }^{(9)}$. For ESBL and AmpC producers, carbapenem remain the drug of choice, whereas in carbapenem resistant strains we are left with Tigecycline and polymyxins which have started developing resistance to many Gram negative bacilli (10). Carbapenem resistance in Acinetobacter may be due to oxacillinases, metallobeta lactamases, AmpC beta lactamases or due to porin deficiency ${ }^{(11)}$. Also metallo beta lactamases are more potent (100-1000 fold) hydrolysers of carbapenems when compared to OXA type carbapenamases which contribute to the carbapenem resistance to a greater extent ${ }^{(12)}$.

Hence the detection of carbapenem resistance is important in the treatment of patients and also preventing the spread of resistant strains, as we have to go a long way for newer antibiotics. The present study was therefore taken to identify the Acinetobacter spp., from various clinical specimens and to detect the MBL production.

To identify, isolate and detect Metallo Beta Lactamase (MBL) resistance in Acinetobacter species and confirmed by phenotypic methods from various clinical samples in a tertiary care hospital

\section{Materials and Methods}

This Cross sectional study was conducted in the Department of Microbiology in a tertiary care hospital over a period of 6 months. Samples were collected from patients attending Out patient Department (OPD) and wards who satisfied the inclusion criteria. Inclusion Criteria included were hospitalized patients of all age groups undergoing treatment in ICU, medical, surgical and paediatric ward, patients affected with burns, Patients with non-healing ulcer, diabetic patients with ulcers, septicemia and pneumonia, peritonitis, patients with indwelling urinary catheter and on ventilators. Exclusion criteria included patients on prior antibiotic therapy, isolates of repeated samples from the same patient, patient who do not give consent.

Isolation and identification is mainly based on the Gram staining, motility, colony morphology on Nutrient Agar, MacConkey Agar and Blood Agar. All the catalase positive, oxidase negative, non-lactose fermenting colonies on MacConkey agar were provisionally identified by colony morphology and biochemical reactions. Acinetobacter species is a Gram negative, non-motile, encapsulated coccobacillus. The colonies which failed to acidify the TSI agar were considered as nonfermenters and subjected to the following tests. Indole, Citrate, Urease, 
Nitrate reduction, growth at $42^{\circ} \mathrm{C}$. Sensitivity to Polymyxin $\mathrm{B}$ and following special biochemical tests and grouped according to Schreckenberger scheme. ${ }^{(1,10)}$

Since there are no CLSI guidelines for the detection of Metallobetalactamase (MBL), different studies used different methods. Despite PCR being highly accurate and reliable, its accessibility is limited only to reference laboratories. The present study was therefore taken to identify the Acinetobacter spp., from various clinical specimens and to determine their antimicrobial susceptibility pattern and also to detect the MBL resistance by different phenotypic methods among Acinetobacter species, in the same isolates.

\section{Antimicrobial susceptibility testing:}

\section{Disc diffusion method}

Antimicrobial susceptibility was performed for all the isolates by modified Kirby -Bauer disc diffusion method. The panel of drugs used for antimicrobial sensitivity testing was as follows: Cefotaxime $(30 \mu \mathrm{g})$, Ceftazidime (30 $\mu \mathrm{g})$, Amikacin $(30 \mu \mathrm{g})$, Gentamycin $(10 \mu \mathrm{g})$, Ciprofloxacin $(5 \mu \mathrm{g})$, Piperazillin /Tazobactum (100/10 $\mu \mathrm{g})$, Trimethoprim/ Sulfamethoxazole $(1.25 / 23.75 \mu \mathrm{g})$, Imipenem $(10 \mu \mathrm{g})$, Meropenem $(10 \mu \mathrm{g})$, Polymyxin B (300U). Interpretations were made using the Clinical and Laboratory Standards Institute, USA guidelines (January 2016, M100-S24Volume 34 No.1, Table 2B-2, Page 62/63) ${ }^{(14)}$. Journal reference was used for Polymyxin B and Colistin Disc diffusion standards as no CLSI guidelines exist for the same. ${ }^{(9,13)}$

\section{Detection of metallo betalactamase production in Acinetobacter spp., by phenotypic methods}

The Acinetobacer isolates which were found to be resistant to Imipenam, Meropenem subjected to various phenotypic detection methods such as Combined disc diffusion Test and Double disc synergy test.

\section{Combined Disc Diffusion Test (CDDT)}

The strain to be tested was inoculated onto MHA plate as suggested by the CLSI. Two $(10 \mu \mathrm{g})$ Imipenem or Meropenem discs were placed on the plate at the distance of $20 \mathrm{~mm}$ and $10 \mu 1$ of $0.5 \mathrm{M}$ EDTA solution was added to one of them to obtain the desired concentration $(750 \mu \mathrm{g})$. After 18 hours of incubation, the increase in inhibition zone with Imipenem EDTA, Meropenem with EDTA disc $\geq 5 \mathrm{~mm}$ than the Imipenem, Meropenem disc alone was considered as MBL positive.

\section{Double Disc Synergy Test (DDST)}

Lawn culture of the test organism was prepared over Mueller-Hinton agar plate as per CLSI guidelines. A plain sterile disc was kept $20 \mathrm{~mm}$ apart from either Imipenem or Meropenem $(10 \mu \mathrm{g})$ disc. $5 \mu \mathrm{l}$ of EDTA was added to plain disc and incubation was done at $37^{\circ} \mathrm{C}$ overnight. Presence of an extended zone from Imipenem or Meropenem disc towards EDTA was interpreted as positive.

\section{Results and Discussion}

All the isolates of Acinetobacter spp., were characterised to the species level and the results were analysed. During the study period, of the 75 Acinetobacter spp., isolated, 50(67\%) were A.baumannii and 25(33\%) were A.lwoffi. Age distribution of Acinetobacter spp., was analysed which showed, majority of the patients were from the age group of more than 50 years of age $21(28 \%)$, followed by $<10$ years $18(24 \%)$ and 21-30yrs $17(23)$ years of age (Table 1). Of the 75 isolates, 46(61\%) were males and 29 (39\%) were females. Majority of isolates of Acinetobacter spp., 
were from Surgical ward (27\%) followed by NICU (20\%). (Table 2). The disk diffusion susceptibility testing of the isolates shows the percentage of sensitivity of the isolates. Among all the isolates maximum resistance was recorded for Gentamycin (64\%), Amikacin, Cotrimoxazole and Ciprofloxacin $(40 \%)$ followed by Cefotaxime and Ceftazidime (36\%) (Table 3). Among the 75 isolates of Acinetobacter spp., screened for Meropenem resistance by Kirby-Bauer disc diffusion method, of which $7(9.3 \%)$ isolates were found to be resistant to Meropenem. Out of 7 isolates of Acinetobacter spp., 5(6.7\%) isolates of A.baumanii and 2(2.6\%) A.lwoffi were MBL producers. Of the $7 \mathrm{MBL}$ resistant strains of Acinetobacter spp., 2 (29\%) were from pus, ET swab and Blood and 1(13\%) from sputum (Table 4). The meropenem resistance by Kirby -Bauer disc diffusion method was taken as the indicator for carbapenamase production and was further tested for their mechanisms of carbapenam resistance confirmed by phenotypic methods.

Among the 7 isolates, CDDT was positive in $5(71 \%)$ isolates, DDST was positive in $3(43 \%)$ isolates. Of the 7 isolates both CDDT, DDST was positive in $3(43 \%)$ isolates and CDDT alone was positive in $4(57 \%)$ isolates (Table 5).

Table.1 Age wise distribution $(n=75)$

\begin{tabular}{|l|c|c|}
\hline \multicolumn{1}{|c|}{ Age in years } & Number of patients & Percentage (\%) \\
\hline $\mathbf{1 0}$ & 18 & 24 \\
\hline $\mathbf{1 1 - 2 0}$ & 3 & 4 \\
\hline $\mathbf{2 1 - 3 0}$ & 17 & 23 \\
\hline $\mathbf{3 1 - 4 0}$ & 8 & 11 \\
\hline $\mathbf{4 1 - 5 0}$ & 8 & 11 \\
\hline $\mathbf{5 0}$ & 21 & 28 \\
\hline TOTAL & 75 & 100 \\
\hline
\end{tabular}

Table.2 Ward wise Acinetobacter spp., isolation ( $\mathrm{n}=75)$

\begin{tabular}{|l|c|c|}
\hline \multicolumn{1}{|c|}{ Ward } & Number of patients & Percentage (\%) \\
\hline NICU & 13 & 20 \\
\hline PICU & 3 & 4 \\
\hline Medicine & 10 & 13 \\
\hline IMCU & 1 & 1 \\
\hline Surgery & 20 & 27 \\
\hline SICU & 1 & 1 \\
\hline O\&G & 7 & 9 \\
\hline Burns & 5 & 7 \\
\hline Urology & 6 & 8 \\
\hline Orthopaedics & 4 & 5 \\
\hline ENT & 3 & 4 \\
\hline Paediatricsward & 2 & 3 \\
\hline TOTAL & 75 & 100 \\
\hline
\end{tabular}

Majority of isolates of Acinetobacter spp., were from Surgical ward (27\%) followed by NICU (20\%). 
Table.3 Antimicrobial susceptibility pattern of Acinetobacter spp., (n=75)

\begin{tabular}{|l|c|c|c|c|}
\hline \multirow{2}{*}{\multicolumn{1}{c|}{ Drugs }} & \multicolumn{2}{c|}{$\begin{array}{c}\text { A.baumaniii } \\
(\mathrm{n}=50)\end{array}$} & \multicolumn{2}{c|}{$\begin{array}{c}\text { A.lwoffii } \\
(\mathrm{n}=25)\end{array}$} \\
\cline { 2 - 6 } & S & $\mathbf{\%}$ & S & $\%$ \\
\hline Gentamycin & 19 & 38 & 9 & 36 \\
\hline Amikacin & 32 & 64 & 15 & 60 \\
\hline Ciprofloxacin & 23 & 46 & 15 & 60 \\
\hline Ceftazidime & 34 & 68 & 16 & 64 \\
\hline Cefotaxime & 34 & 68 & 16 & 64 \\
\hline Pip - Taz & 32 & 64 & 17 & 68 \\
\hline Cotrimoxazole & 10 & 20 & 15 & 60 \\
\hline Imipenem & 45 & 90 & 23 & 92 \\
\hline Meropenem & 45 & 90 & 23 & 92 \\
\hline Polymyxin - B & 50 & 100 & 25 & 100 \\
\hline
\end{tabular}

The disk diffusion susceptibility testing of the isolates shows the percentage of sensitivity of the isolates. Among all the isolates maximum resistance was recorded for Gentamycin (64\%), Amikacin, Cotrimoxazole \&

Ciprofloxacin( $40 \%)$ followed by Cefotaxime \& Ceftazidime (36\%).

Table.4 Sample distribution of MBL isolates ( $\mathrm{n}=7$ )

\begin{tabular}{|l|c|c|}
\hline Clinical samples & No. of MBL & Percentage (\%) \\
\hline Pus & 2 & 29 \\
\hline ET swab & 2 & 29 \\
\hline Sputum & 1 & 13 \\
\hline Blood & 2 & 29 \\
\hline Total & 7 & 100 \\
\hline
\end{tabular}

Of the 7 MBL resistant strains of Acinetobacter spp., 2 (29\%) from pus, ET swab and Blood and 1(13\%) from sputum

Table.5 Comparison of MBL detection by different methods

\begin{tabular}{|l|c|c|c|c|c|}
\hline \multirow{2}{*}{ Organism } & \multirow{2}{*}{ No. } & \multicolumn{2}{c|}{$\begin{array}{c}\text { Double disc } \\
\text { Synergy test }\end{array}$} & \multicolumn{2}{c|}{$\begin{array}{c}\text { Combined disc } \\
\text { test }\end{array}$} \\
\cline { 3 - 6 } & & $+\mathbf{v e}$ & -ve & + ve & - ve \\
\hline A.baumanii & 5 & 3 & 2 & 5 & - \\
\hline A.lwoffii & 2 & - & 2 & 2 & - \\
\hline Total & 7 & 3 & 4 & 7 & \\
\hline
\end{tabular}


Fig.1 Age wise distribution $(\mathrm{n}=75)$

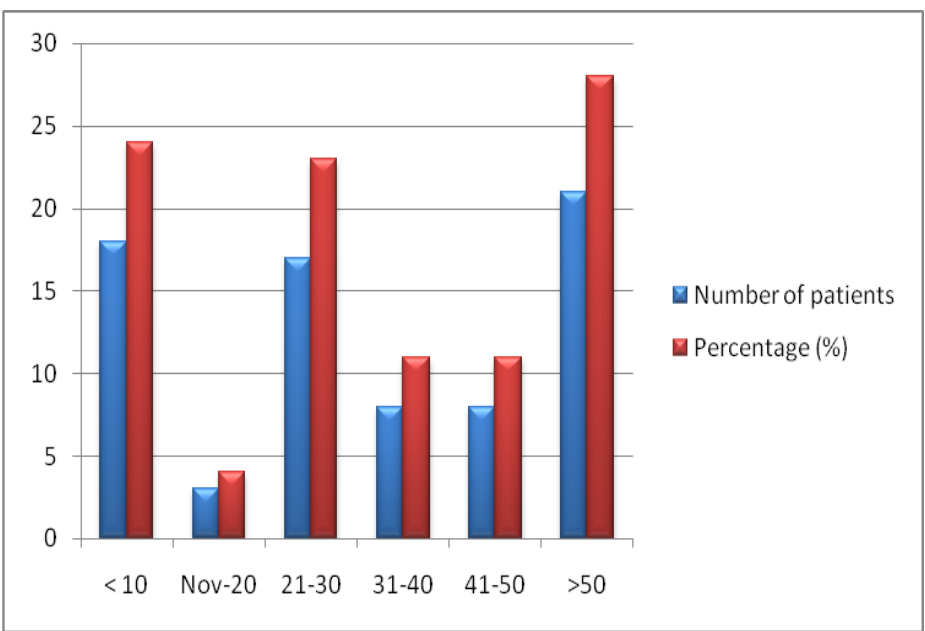

Age distribution of Acinetobacter spp., was analysed which showed, majority of the patients were from the age group of more than 50 years of age $21(28 \%)$, followed by $<10$ years $18(24 \%)$ and $21-30$ yrs $17(23)$ years of age.

Fig.2 Gender distribution $(\mathrm{n}=75)$

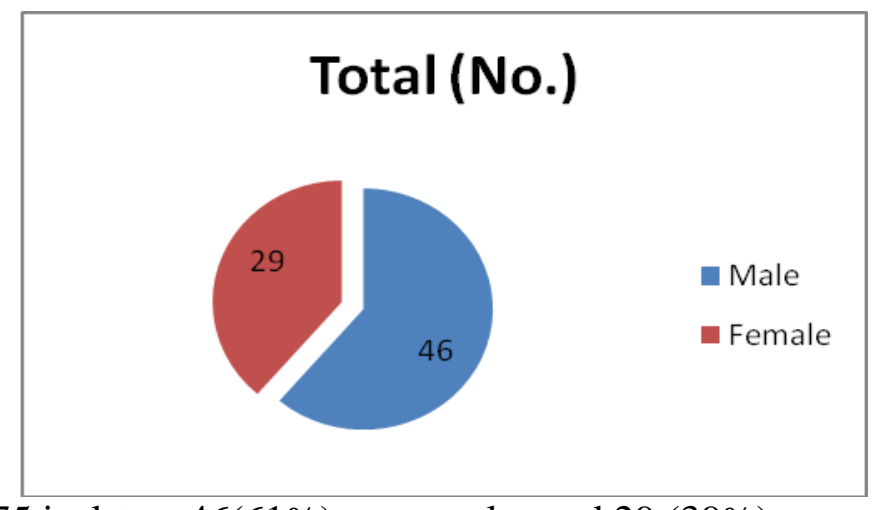

Of the 75 isolates, 46(61\%) were males and $29(39 \%)$ were females.

Fig.3 Ward wise Acinetobacter spp., isolation $(\mathrm{n}=75)$

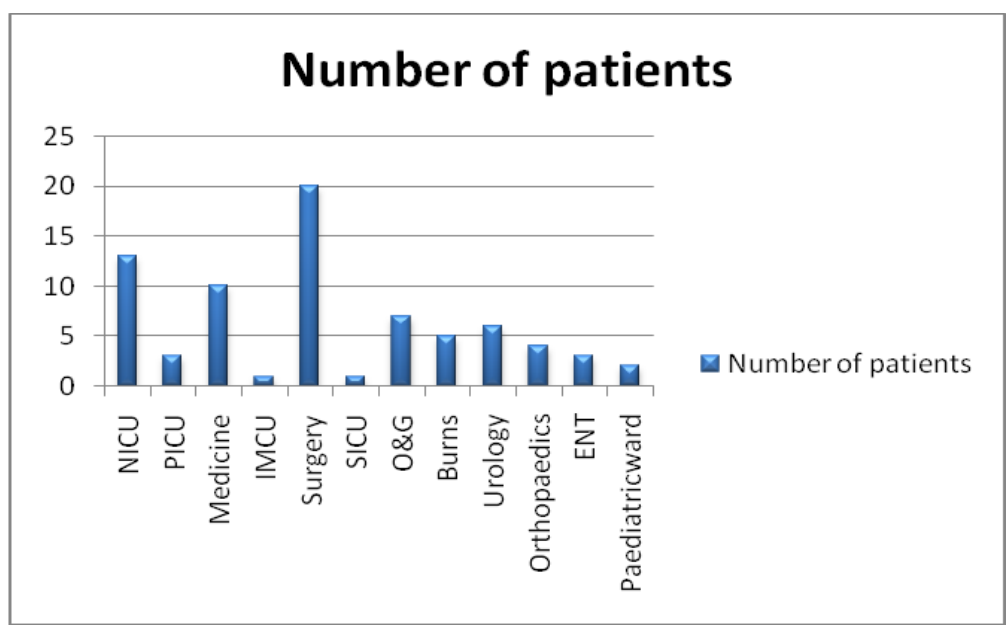


Fig.4 Sample distribution of MBL isolates $(n=7)$

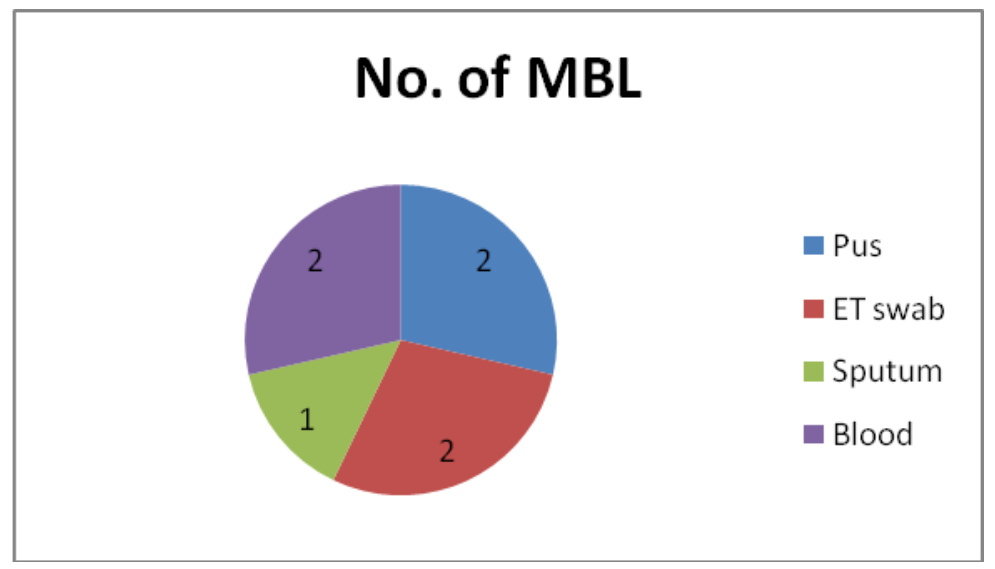

Imipenem-EDTA combined disc test for MBL detection

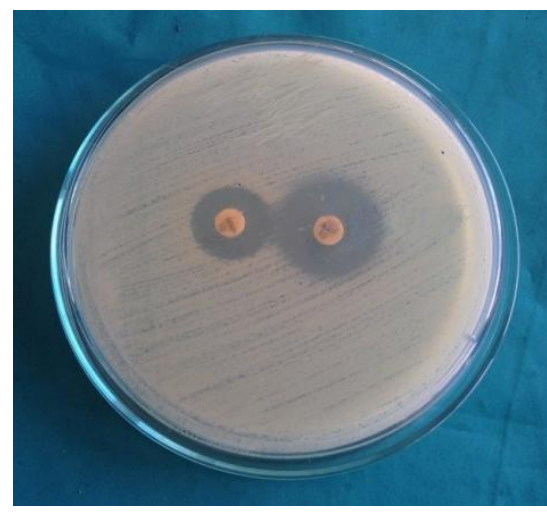

I - Imipenem IE - Imipenem EDTA

Double disk synergy test for MBL detection

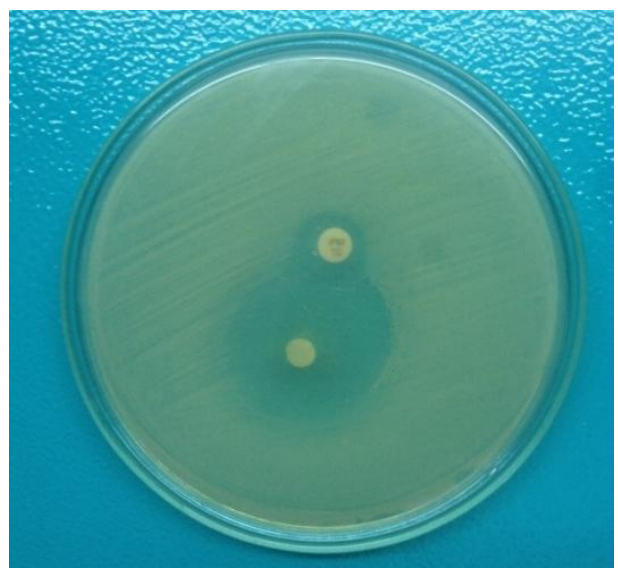


The meropenem resistance by Kirby -Bauer disc diffusion method was taken as the indicator for carbapenamase production and was further tested for their mechanisms of carbapenam resistance confirmed by phenotypic methods.

Among the 7 isolates, CDDT was positive in $5(71 \%)$ isolates, DDST was positive in $3(43 \%)$ isolates. Out of the 7 isolates CDDT, DDST was positive in $3(43 \%)$ isolates and CDDT alone was positive in $4(57 \%)$ isolates.

Non fermenting Gram Negative Bacilli (NFGNB) are being isolated with increasing frequency from clinical specimens and treatment failure due to their multidrug resistance in the recent years. In our study, we have isolated 75 Acinetobacter spp., over a period of 6 months from various clinical samples and were evaluated for their role in infections in hospitalized patients including the drug resistance and screening for MBL production revealed that $7(9.3 \%)$ isolates were found to be resistant to Meropenem. The prevalence and sensitivity of nonfermenters often varies between communities among different patient populations in the same hospital. Faced these variations, the physician in the clinical practice has the responsibility to acess recent data on the prevalence and resistance pattern of commonly encountered pathogens ${ }^{(15)}$.

The present study observed highest resistance of Acinetobacter spp., against first line antibiotics which are the commonly used drugs. This necessitates the judicious use of these antibiotics in empirical therapy. Maximum sensitivity was observed with newer agents like carbapenams and pipercillin-tazobactum and Polymyxin, Moderatly sensitive to Aminoglycosides and Fluroquinolones. Major risk of using monotherapy is the emergence of antibiotic resistance as observed in the present study which showed high rate of multidrug resistance and MBL producers.

Carbapenamase resistance was observed as emerging drug resistant mechanisms in the NFGNB from this hospital. Antibiotic therapy either empirical or documented is based upon antibiotic combination supplemented by the knowledge of local epidemiology of susceptibility pattern in choosing a suitable combination. Therefore combination therapy such as piperacillin-tazobactum, quinolones amikacin, imipenam-amikacin would be an ideal choice of therapy on the basis of antimicrobial susceptibility testing as observed in this study along with an adequate infection control measures especially in the surgical and ICU units. ${ }^{(16,17)}$

The treatment of Acinetobacter infections remains a great challenge because resistance to aminoglycosides, cephalosporins and quinolones has substantially increased worldwide. Carbapenems are the drug of choice for MDR Acinetobacter infections, for ESBL producing isolates, but resistance to carbapenems by the production of carbapenamases and various other mechanisms has limited the therapeutic options. ${ }^{(18)}$ Because of increasing carbapenem resistance and limited therapeutic options available, the old antibiotic colistin is being used more extensively nowadays, but resistance to colistin has also been reported. ${ }^{(19)}$. In my study all the isolates were sensitive to Polymyxin B. Hence currently combination therapy like meropenem with tigecycline and colistin with sulbactum or rifampicin are being tried in the treatment of Acinetobacter spp., infection ${ }^{(20)}$.

The prevalence and sensitivity of nonfermenters often varies between communities. Faced these variations, the physician in clinical practice has the responsibility of making clinical judgments and should access to recent data on the 
prevalence and antimicrobial resistance pattern of commonly encountered pathogens. It is therefore important to institute a system for the surveillance of antimicrobial resistance that will involve the collection of both clinical and microbiological data. Difference in antimicrobial susceptibility poses a great problem in treating these infections. Multidrug resistance by these organisms leads to high morbidity and mortality and left with the only option of treating them by potentially toxic drugs like Colistin and Polymyxin B. This warrants the judicious use of antimicrobial drugs after appropriate laboratory screening and confirmatory methods.

\section{References}

1. Koneman EW,Allen SD. Colour atlas and Text book of diagnostic Microbiology, 6th edition, Philadelphia, Lippincott-Williams\& Wilkins Publishers,2006:p.624-662.

2. Gokhale S, Metgud SC. Characterization and antibiotic sensitivity pattern of nonfermenting Gram negative bacilli from various clinical samples in a tertiary care hospital. Belgaum J Pharm Biomed Sci 2012;17:1-5.

3. Kirtilaxmi K. Benachinmardi, Padmavathy M, Malini J, Naveneeth B. V Prevalence of non-fermenting Gram-negative bacilli and their in vitro susceptibility pattern at a tertiary care teaching hospital. Department of Microbiology, Employees' State Insurance Corporation Medical College and PGIMSR, Bengaluru,Karnataka, India.

4. Quinn JP. 1998 Clinical problems posed by multiresistant nonfermenting gram negative pathogens. Clin Infect Dis. Suppl 1:S117-24.

5. Mandell, Douglas and Bannett's.
Principles \& Practice of Infectious Diseases. 7thEdition, p. 2881-84.

6. Sinha N, Agarwal J, Srivastava S, Singh M. Analysis of Carbapenemresistant Acinetobacter from a tertiary care setting in North India. Indian J Med Microbiol.2013;31:p.60-3.

7. Fellpe Fernandez, Luis Martinez, M Carman, Juan A. Relationship Between Betalactamase production, Outer membrane proteins and Penicillin binding proteins profiles on the activity of Carbapenems against clinical isolates of A.baumannii. J. Antimicrob. Chemo. 2003;51(3):p.565-74.

8. The Genus Acinetobacter Prokaryotes, 2006(6), Towner

9. Gales AC, Reis AO, Jones RN Contemporary assessment of antimicrobial susceptibility testing methods for polymyxin B and colistin: review of available interpretative criteria and quality control guidelines. J Clin Microbiol. 2001 Jan;39(1):18390.

10. Taneja N, singh G, Singh M, Sharma M. Emergence of Tigecycline and colistin resistant A.baumannii in patients with complicated UTI in North India. Indian $\mathrm{J}$ Med res 2011;133:p.681-684.

11. M Sinha H Srinivasa. Mechanisms of resistance to carbapenems in meropenem resistant Acinetobacter isolates from clinical samples. Indian J Med Microbiol. 2007;25(2):121-5.

12. Gomathy Mahajan, Sheevani Sheemar, Shashi Chopra et al.Carbapenem Resistance and Phenotypic Detection of Carbapenamases in Clinical isolates of A.baumannii.Indian $\mathrm{J}$ of Med sciences 2011;65(1):18-25.

13. Galani I, Kontopidou F, Souli M, Rekatsina PD et al. Colistin 
susceptibility testing by E test \& Disc Diffusion methods. Int J Antimicrobial Agents 2008; 31: 434-9.

14. CLSI document M100-S 24.vol.34 No.1. Performance standards for Antimicrobial Susceptibility Testing; 24 informational supplements. Jan14, Interpretive standards for Pseudomonas spp. Acinetobacter spp., S. maltophilia, B. cepacia.

15. Dheepa Muthusamy \& Appalraju Boppe. Phenotypic Methods for the detection of various Betalactamases in Carbapenamase resistant isolates of A.baumannii in a Tertiary care Hospital in a south India. India. $J$ of Clin Diagnostic Research. 2012;6(6):p.970-73

16. Veenu, Rama S, Arora DR. 1999 Isolation and susceptibility pattern of non fermenting Gram negative bacilli from clinical samples. Indian $\mathrm{J}$ Med Microbiol;17(1):14-7.
17. Vijaya D, K. Bavani S, Veena M, 2000 Prevalence of nonfermenters in clinical specimens:54:87-91 Indian journal of medical sciences volume54, Issue 3, p87-91.

18. Yang Soon Lee, Young Ree Kim et al. Increasing prevalence of blaOXA-23 carrying A.baumannii and the emergence of blaOXA-182 carrying A. nosocomialis in Korea. Diag. Microbiol \& Infectious Disease2013; 7(2): 160- 63.

19. Nasrollah Sohrabi et al. Prevalence of OXA type beta lactamases among $A$. baumannii isolates from North west of Iran. Microbiol Drug Resistance 2012;18(4):385-9.

20. Roxanne J Owen, Jian Li, Roger L Nation, Devis Sperman. Invitro Pharmacodynamics of Colistin against A.baumannii clinical isolates. $\mathrm{J}$ of Antimicrobial Chemotherapy. 2006; 59(3): 473-77.

\section{How to cite this article:}

Sundararajan Thangavel, Gomathi Manian, Neelaveni Ramasamy 2018. Identification, Isolation and Detection of Metallo Beta Lactamase Resistance in Acinetobacter Species from Various Clinical Samples in a Tertiary Care Hospital Int.J.Curr.Microbiol.App.Sci. 7(07): 2378-2387. doi: https://doi.org/10.20546/ijcmas.2018.707.277 